\title{
Link-Adaptive Largest-Weighted-Throughput Packet Scheduling for Real-Time Traffics in Wireless OFDM Networks
}

\author{
Ying Jun (Angela) Zhang, Member, IEEE and Soung Chang Liew, Senior Member, IEEE \\ Department of Information Engineering \\ The Chinese University of Hong Kong, Shatin, New Territories, Hong Kong \\ yjzhang@ie.cuhk.edu.hk, soung@ie.cuhk.edu.hk
}

\begin{abstract}
The explosive growth of high-data-rate and multimedia real-time applications imposes new challenges on the design of future wireless communications systems. In this paper, a cross MAC-PHY layer link-adaptive largest-weighted-throughput packet scheduling algorithm is proposed to provide QoS guarantees to real-time traffics over wireless packet-switched networks. The proposed algorithm aims to satisfy the stringent packet delay constraints while obtaining a high system spectral efficiency. The objective is achieved by two interactive components: a MAC service discipline and a PHY rate-and-power adaptation scheme. It is demonstrated in the numerical results that the proposed algorithm significantly improves the system performance in terms of spectral efficiency and packet loss rate, thanks to the successful exploitation of the inherent system diversities in the time, frequency, and multiuser domains.
\end{abstract}

Key Words-Opportunistic scheduling, Link adaptation, OFDM.

\section{INTRODUCTION}

The next generation wireless systems are expected to provide a wide range of high-data-rate services, including non-real-time data applications and real-time voice or video applications. Providing QoS (Quality of Service), in particular meeting the data rate and packet delay constraints of the real-time flows, is one of the most challenging requirements in future wireless communications systems.

Packet scheduling has long been considered as an important mechanism to ensure the QoS in wired networks [1]. Recently, new schemes for packet scheduling in wireless networks have emerged, wherein the unique characteristics of wireless media such as multipath fading, high error rate, and time-varying channel capacity are considered [2]. The objectives of these schemes are to maximize the system spectral efficiency by exploiting the random channel variation, and/or to provide fairness and QoS guarantees to the users by deferring the transmissions on the links that are in deep fading and compensating for the transmissions when the link qualities recover [3-8].

However, most of the existing QoS scheduling schemes are able to provide short-term fairness and delay/throughput guarantees only to the users whose channels are temporarily in error-free states [2]. Consequently, these schemes fail to support real-time services over wireless media, especially when the channel varies slowly compared to the delay constraints of the applications. Besides, a majority of the existing packet scheduling schemes assume either a binary channel model $(0 \%$ or $100 \%$ link error) $[3,4,8]$ or a flat fading channel model $[6,7]$. In fact, neither of the models is realistic in high-data-rate and broadband wireless systems.
In this paper, we propose a cross-layer link-adaptive largest-weighted-throughput (LWT) scheduling algorithm for real-time applications under a realistic continuous and frequency-selective fading channel model. The proposed algorithm aims to satisfy the stringent packet delay constraints for each user, while maximizing the system spectral efficiency over the downlink. This objective is achieved by two mutually cooperative parts: MAC-layer opportunistic scheduling and PHY-layer link adaptation. The MAC scheduling scheme schedules transmissions according to the packet delay constraints and the attainable throughputs of each link to ensure timely and spectrum-efficient delivery of the packets. Meanwhile, the PHY link adaptation scheme dynamically adjusts the transmission rate and power to maximize the per-link throughput, maintain the PER (packet error rate) at a sufficiently low level, and make the wireless channel appear to be almost error free to the upper layers. In addition, OFDM (Orthogonal Frequency Division Multiplexing) is adopted as the air interface due to its excellent performance of combating the multipath delay spread in frequency selective fading channels [10].

Thanks to the cooperation between the MAC and PHY layers, the proposed algorithm outperforms the traditional packet scheduling algorithms in the following aspects. 1) Most of the existing scheduling schemes defer the transmissions of the users who are in deep fading, and hence fail to satisfy the stringent delay constraints of real-time packets, especially when the channel varies slowly. In contrast, the proposed scheduling scheme endeavors to guarantee timely deliveries of the packets regardless of the underlying channel conditions. A high probability of successful packet reception (i.e., a low PER) is ensured by the PHY-layer link adaptation, even if the packet is transmitted over an unfavorable channel. 2) The proposed algorithm improves the spectral efficiency by giving higher priorities to the packets that are to be transmitted over the links with higher attainable throughputs. Compared with the existing opportunistic scheduling schemes [5-7], the proposed scheme achieves much higher spectral efficiency due to the exploitation of channel variations in the time, frequency, and multiuser domains. 3) In addition and in contrast to the previous work, realistic throughputs (which are affected by instantaneous channel coefficients, PER requirements, transmission power constraints, and the type of constellation adopted) instead of channel capacity is considered in our scheduling rule.

The remainder of this paper is organized as follows. The system model of the proposed algorithm is introduced in the next section. In Section III, the link-adaptive LWT scheduling algorithm is presented. The performance of the proposed algorithm is investigated and compared with traditional scheduling schemes in Section IV. Finally, the 
paper is concluded in Section V.

\section{SYSTEM MODEL}

This paper investigates the downlink transmission of a packet-switched network with OFDM signaling. As in the previous work [3-8], CSI (Channel State Information) is assumed to be available at the AP (Access Point). Assume that the frequency bandwidth is divided into $N$ subcarriers and there are totally $K$ active users. The AP is in charge of packet scheduling and resource allocation, as shown in Fig. 1. Upon arriving at the AP, the packets intended for different users are buffered in separate queues. For simplicity, packets are assumed to have equal length and contain $L$ bits each. Once the channel becomes idle, a HOL (Head Of Line) packet is selected for transmission according to the proposed LWT service discipline. Meanwhile, a link-adaptation scheme is applied to determine the optimal rate-and-power allocation over the subcarriers of each OFDM symbol based on the instantaneous channel conditions. According to the rate-and-power allocation decision, the selected packet is demultiplexed and modulated onto the $N$ subcarriers. After IFFT (Inverse Fast Fourier Transform), parallel/serial conversion, and adding cyclic prefix, the packet is transmitted via the downlink channel. Assume that the channel varies relatively slowly and the length of the cyclic prefix is longer than the maximum time dispersion. Consequently, ISI (InterSymbol Interference) is perfectly mitigated and the channel appears to be flat on every subcarrier. Let us denote the channel coefficient seen by user $k$ on subcarrier $n$ during the $s^{\text {th }}$ OFDM symbol by $h_{k, n}(s)$. Likewise, the bit and power allocation on subcarrier $n$ during OFDM symbol $s$ if the selected packet is intended for user $k$ is denoted by $b_{k, n}(s)$ and $p_{k, n}(s)$, respectively.

In this paper, we are interested in real-time traffic for which packets must be delivered to the destinations before certain delay upper bounds. ARQ (Automatic Return reQuest) is applied to recover the packet errors, and a packet is retransmitted until it is received successfully or is dropped due to the expiration of the deadline. Obviously, the transmission efficiency, which is defined to be the ratio of the number of successfully transmitted packets to the total number of packet transmissions, is highly dependent of the PER, denoted by $\varepsilon_{p}$. In order to achieve a sufficiently low PER, $b_{k, n}(s)$ should be selected according to the target value of $\varepsilon_{p}$, the transmission power $p_{k, n}(s)$, and the instantaneous channel coefficient $h_{k, n}(s)$. That is,

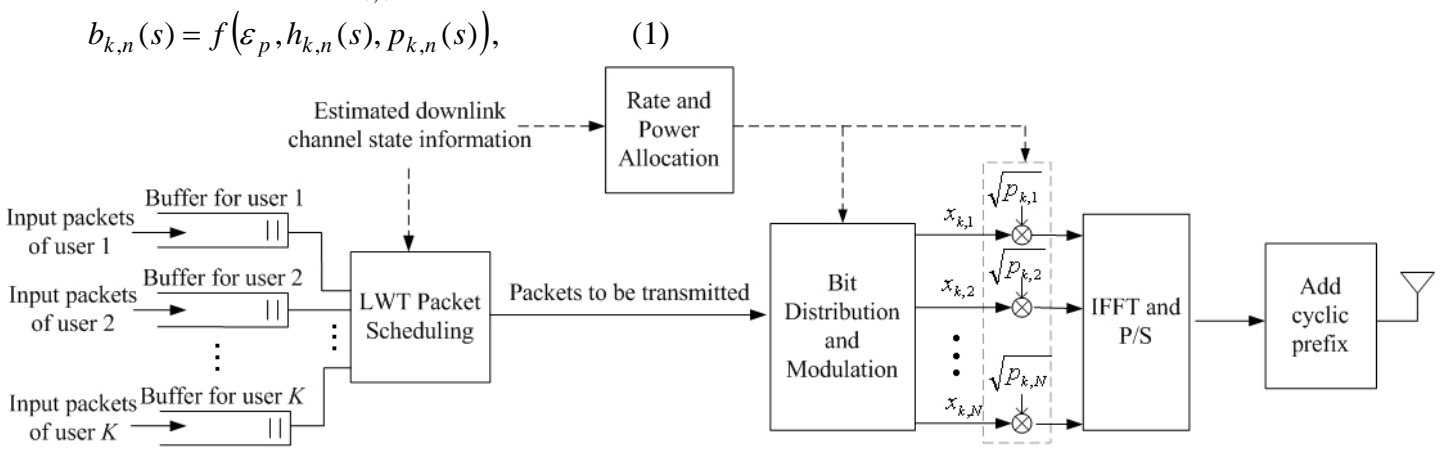

Fig. 1: Transmitter Structure at the Access Point where $f(\cdot)$ is a nonlinear function depending on the type of constellation adopted. Moreover, if a limit of the maximum transmission power is present, the following equation

$$
\sum_{n=1}^{N} p_{k, n}(s) \leq P_{T}
$$

should be satisfied, where $P_{T}$ is the upper bound of the total transmission power in one OFDM symbol.

\section{LINK-ADAPTIVE LWT PACKET SCHEDULING}

In this section, we describe the proposed link-adaptive LWT scheduling algorithm. First, the optimal solution for PHY rate-and-power allocation is derived, based on which we can calculate the maximum attainable throughput for each downlink channel. Then, the MAC scheduling scheme that schedules the packet transmissions according to a largest-weighted-throughput criterion is presented.

\subsection{PHY Rate-and-Power Adaptation}

The objective of the rate-and-power adaptation is to find for each user $k$ and OFDM symbol $s$ the best assignment of $b_{k, n}(s)$ and $p_{k, n}(s)$ such that the per-link throughput (defined to be the number of bits transmitted per OFDM symbol) given by

$$
T_{k}(s)=\sum_{n=1}^{N} b_{k, n}(s)
$$

is maximized subject to a power constraint $P_{T}$ and a PER constraint specified through $f(\cdot)$. At high SNR (Signal to Noise Ratio), the expression of BER (bit error rate) for square MQAM (M-ary Quadrature Amplitude Modulation) or MPSK (M-ary Phase Shift Keying) with Gray bit mapping as a function of the received SNR is approximated as [11]

$$
\varepsilon_{b} \approx c_{1} \exp \left(\frac{-c_{2} p_{k, n}(s)\left|h_{k, n}(s)\right|^{2} / \sigma^{2}}{2^{c_{3} b_{k, n}(s)}-1}\right),
$$

where $\sigma^{2}$ denotes the variance of noise, and $c_{1}, c_{2}$, and $c_{3}$ are positive fixed constants. If we assume that all bits are independent and are equally protected in a packet, then PER can be written as a function of BER:

$$
\varepsilon_{p}=1-\left(1-\varepsilon_{b}\right)^{L} \approx L \varepsilon_{b} .
$$


Thus, the nonlinear function $f(\cdot)$ in Eqn. (1) becomes

$$
b_{k, n}(s)=\frac{1}{c_{3}} \log _{2}\left(1+\frac{c_{2} p_{k, n}(s)\left|h_{k, n}(s)\right|^{2} / \sigma^{2}}{\ln \left(L c_{1} / \varepsilon_{p}\right)}\right) .
$$

Now, the optimal rate and power allocation that maximizes the throughput (3) subject to the power constraint (2) and PER constraint (6) is readily derived using the Lagrange method. For user $k$ and OFDM symbol $s$, the optimal allocation solution is

$$
\begin{aligned}
& p_{k, n}^{*}(s)= \\
& \left\{\begin{array}{c}
\lambda_{k}(s)-\frac{\sigma^{2} \ln \left(L c_{1} / \varepsilon_{p}\right)}{c_{2}\left|h_{k, n}(s)\right|^{2}} \quad \frac{\left|h_{k, n}(s)\right|^{2}}{\sigma^{2}} \geq \frac{\ln \left(L c_{1} / \varepsilon_{p}\right)}{c_{2} \lambda_{k}(s)} \\
0
\end{array} \quad\right. \text { else }
\end{aligned}
$$

and

$$
\begin{aligned}
& b_{k, n}^{*}(s)=
\end{aligned}
$$

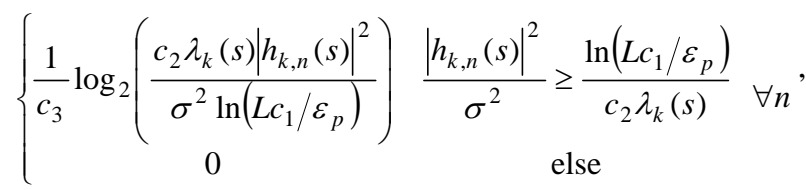

where $\lambda_{k}(s)$ is a constant such that the total power constraint (2) is satisfied.

So far, we have assumed a continuous rate adaptation, i.e., $b_{k, n}(s)$ is a continuous nonnegative variable. In realistic systems, however, only a discrete finite set of $J$ constellations is available. That is, $b_{k, n}(s)$ is restricted to a fixed set of nonnegative integer values $\left\{b_{j}\right\}_{j=0}^{J-1}$. With this additional constraint, no closed-form solution to the optimization problem is available. In this case, the optimal rate-and-power adaptation can be achieved by a greedy algorithm, which initially assigns zero bits to all subcarriers and then allocates bit by bit to the subcarrier that requires the least additional transmission power. The allocation is repeated until the upper bound of the total transmission power is reached.

Obviously, a discrete rate adaptation yields a lower throughput than a continuous rate adaptation due to the additional constraint. That is,

$$
\hat{T}_{k}(s)=\sum_{n=1}^{N} \hat{b}_{k, n}(s) \leq \sum_{n=1}^{N} b_{k, n}^{*}(s) \forall k, s,
$$

where $\hat{b}_{k, n}(s)$ denotes the optimal rate allocation in a discrete case. Therefore, $\sum_{n=1}^{N} b_{k, n}^{*}(s)$, which can be calculated numerically, can be used as an upper bound of the maximum achievable throughput in a realistic system with discrete rate adaptations.

\subsection{MAC LWT Packet Scheduling}

The scheduling scheme endeavors to solve the following problem: given the per-link throughput $\hat{T}_{k}(s)$, the waiting time $w_{k}$ of the HOL packets, and the delay constraints $d_{k}$ of the applications, which user should be served so that the system spectral efficiency is maximized and the delay constraints of the packets are satisfied. Since the packets are assumed to be of equal length, the spectral efficiency can be measured by the time needed to send one packet. In our system, the transmission time is expressed in terms of the number of OFDM symbols. That is,

$$
Q_{k}=\min \left\{q \mid \sum_{s=1}^{q} \hat{T}_{k}(s) \geq L\right\} \forall k,
$$

where $Q_{k}$ denotes the transmission time of a packet in queue $k$. Obviously, the smaller the value of $Q_{k}$, the larger the spectral efficiency. On the other hand, the remaining time to the deadline of the HOL packet in queue $k$ is calculated as

$$
r_{k}=d_{k}-w_{k} \forall k \text {. }
$$

The smaller the value of $r_{k}$, the more urgent it is to transmit the corresponding packet. It is also noted that when $r_{k}<Q_{k}$, transmitting the packet causes a waste of bandwidth, because the delay upper bound is inevitably violated by the end of the transmission. Therefore, the packet should be temporarily excluded from scheduling. The scheduling may recover later when the channel condition of user $k$ becomes better and $Q_{k}$ becomes smaller.

Our scheduling rule is described as follows. Once the channel is idle, the HOL packet with the minimum value of

$$
Q_{k} r_{k} \frac{1}{\alpha} \delta\left(r_{k}-Q_{k}\right)
$$

is scheduled for transmission. In (12), $\delta(\cdot)$ is defined as

$$
\delta(x)= \begin{cases}1 & x \geq 0 \\ \infty & x<0\end{cases}
$$

to ensure that the packet is excluded from scheduling when $r_{k}<Q_{k} . \alpha$ is a positive constant to adjust the weight of the effect of $r_{k}$ relative to $Q_{k}$.

Typically, the channel coherence time is much longer than the transmission period of a packet in an indoor WLAN environment. It is therefore reasonable to assume that $h_{k, n}(s)$ does not change during the transmission of a packet. Hence, the same optimal rate-and-power allocation solution applies to all the OFDM symbols in a packet. It follows immediately that

$$
\begin{gathered}
\hat{T}_{k}(s)=\hat{T}_{k} \forall s=1, \cdots, Q_{k}, \\
Q_{k}=\left\lceil\frac{L}{\hat{T}_{k}}\right\rceil,
\end{gathered}
$$

and the packet selection criterion becomes

$$
\tilde{k}=\underset{k}{\arg \min } Q_{k} r_{k}{ }^{\frac{1}{\alpha}} \delta\left(r_{k}-Q_{k}\right) \approx \underset{k}{\arg \max } \frac{\zeta\left(r_{k}-L / \hat{T}_{k}\right)}{r_{k}{ }^{\frac{1}{\alpha}}} \hat{T}_{k},
$$

where

$$
\zeta(x)=\left\{\begin{array}{ll}
1 & x \geq 0 \\
0 & x<0
\end{array} .\right.
$$

The scheduling rule described in Eqn. (16) is referred to as Largest-Weighted-Throughput, since the priority of each HOL packet is given by its throughput weighted by a function of the waiting time and the delay constraints. This 
rule combines the merits of the pure opportunistic scheduling and EDD (Earliest Due Date) scheduling schemes. On the one hand, a timely delivery of the packets is ensured by assigning high priorities to the packets whose deadlines are about to expire (indicated by a small $r_{k}$ ). If the intended user happens to experience an unfavorable channel, a good reception quality is guaranteed by the PHY rate-and-power adaptation scheme at the price of possibly reducing the spectral and power efficiency. On the other hand, the scheduling rule improves the spectral/power efficiency by assigning higher priorities to the users with larger throughputs $\hat{T}_{k}$. The tradeoff between resource efficiency and delay bound guarantee is balanced by choosing an appropriate $\alpha$. In addition, the function $\zeta(\cdot)$ avoids wasting the resources on the channels with too low capacities.

The scheduling scheme in (16) is practically difficult to implement since $\hat{T}_{k}$ cannot be calculated numerically. Fortunately, the attainable throughputs for both continuous and discrete rate adaptation are very close to each other when the cardinality of the set of the constellations is large enough [9]. Therefore, the scheduling can be simplified by replacing $\hat{T}_{k}$ with $\sum_{n=1}^{N} b_{k, n}^{*}$ in (16). Thus, the packet selection criterion becomes:

$$
\tilde{k}=\underset{k}{\arg \max } \frac{\zeta\left(r_{k}-L / \sum_{n=1}^{N} b_{k, n}^{*}\right)}{r_{k}^{\frac{1}{\alpha}}} \sum_{n=1}^{N} b_{k, n}^{*},
$$

where $b_{k, n}^{*}$ can be calculated from (7) and (8).

\section{NUMERICAL RESULTS}

In this section, the performance of the proposed link-adaptive LWT scheduling algorithm is investigated and compared with various existing packet scheduling algorithms for wireless networks. A frequency-selective Rayleigh fading channel with an exponential delay profile is considered. Assume that each flow generates packets at an average rate of $100 \mathrm{kbps}$. Fixed length packets of 1000 bytes arrive at the AP according to a Poisson process. The delay upper bound requirement is set to $100 \mathrm{~ms}$. The frequency band is divided into 64 subcarriers and OFDM symbol duration is equal to $200 \mu \mathrm{s}$. Assume that 5 different constellations are available for discrete rate adaptation, i.e., QPSK, 8QAM, 16QAM, 32QAM, and 64QAM.

In the following figures, we compare the performance of the proposed system with four widely accepted wireless service disciplines. They are CSD (Channel State Dependent) RR (Round Robin) [12], FEDD (Feasible EDD) [13], PO (Pure Opportunistic), and M-LWDF (Modified Largest Weighted Delay First) [6] service disciplines. For CSD RR and FEDD schemes, the channel is considered to be in an error state if

$$
\sum_{n=1}^{N}\left|h_{k, n}\right|^{2} / N<0.8
$$

and in an error-free state otherwise. Typically, no link adaptation is adopted in these two schemes, and hence we adopt a fixed modulation of 16QAM in the simulations. Likewise, the PO scheduler schedules transmission corresponding to the user with the highest downlink channel capacity $C_{k}$, while the M-LWDF scheduler adopts the following packet selection criterion [6]:

$$
k^{\prime}=\underset{k}{\arg \max }\left(w_{k} C_{k}\right) \text {. }
$$

Adaptive modulation is adopted in the PHY layer for both the PO and M-LWDF schemes.

In Fig. 2, the average spectral efficiencies are plotted as a function of average transmission power when there are 10 active users in the system. At the high SNR region, the system service rate is higher than the packet arrival rate, and thus the spectral efficiency is mainly determined by the amount of input traffic. It can be seen from the figure that the spectral efficiency is significantly enhanced by the proposed cross-layer algorithm. It is increased by around $17 \%$ compared to the PO scheduler and over 100\% compared to the CSD-RR, FEDD, and LWDF schedulers.

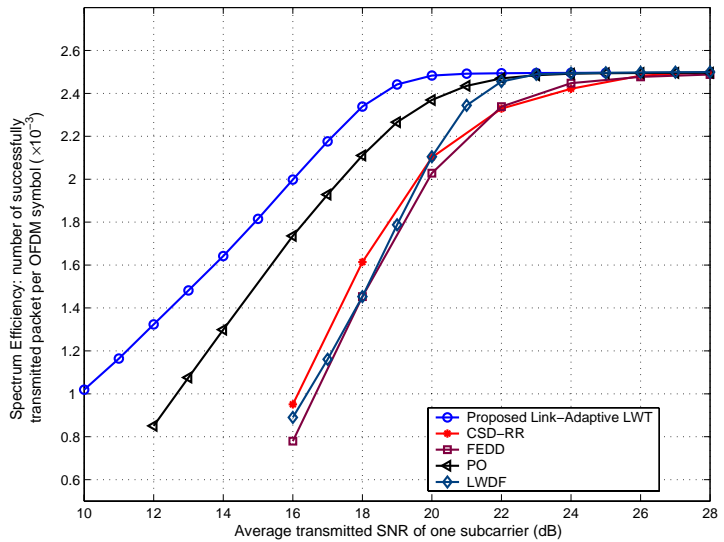

Fig. 2: Comparison of the spectral efficiencies between the link-adaptive LWT algorithm and the traditional service disciplines

Higher spectral efficiency by itself does not necessarily imply a better performance in real-time applications. Packet losses due to the violations of the maximum delay constraints sometimes dominate the service quality. Fig. 3 investigates the packet loss rates of the scheduling schemes. It can be seen that without adaptive modulation, the attainable throughput of each link is fixed and the packet loss rates of the CSD-RR and FEDD are lower-bounded in the high SNR region. The lower bounds are given by the packet loss rates of their wired counterparts with error-free channels (i.e., RR and EDD). In contrast, the attainable throughput increases with SNR when the modulations are adaptive in the PHY layer. Therefore, no "floors" in the packet loss rates are observed for the link-adaptive LWT, PO, and LWDF schemes. The figure also shows that in addition to a higher spectral efficiency, the proposed algorithm is able to achieve a dramatically lower packet loss rate. A 3 4dB power gain over the PO and LWDF schedulers is observed at packet loss rate $10^{-4}$, for instance. The improvement is due to the appropriate packet selection criterion as well as the successful exploitation of multiuser diversity and channel 
variations in the time and frequency domains.

The advantage of the proposed algorithm is further illustrated in Fig. 4 where the packet loss rate is plotted as a function of the number of active users. Similar to the above figures, significant performance improvement of the proposed algorithm is observed. About 3 to 5 more users can be supported compared to the traditional scheduling schemes when packet loss rate is $10^{-2}$, for instance.

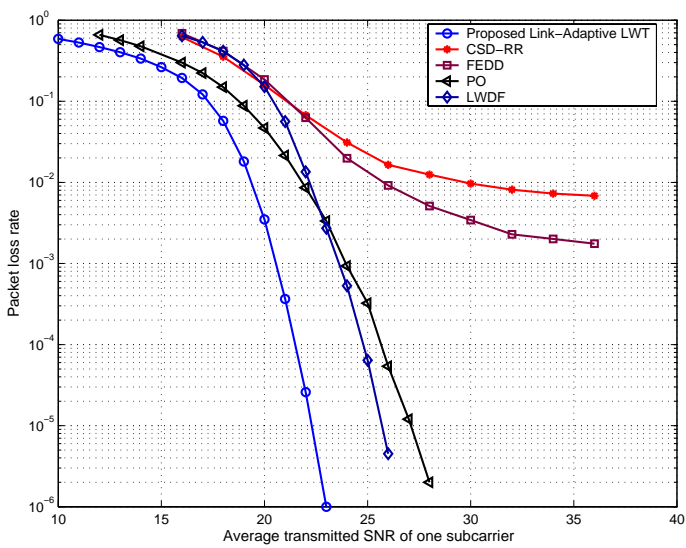

Fig. 3: Comparison of packet loss rate between the proposed link-adaptive LWT scheduling algorithm and the traditional service disciplines

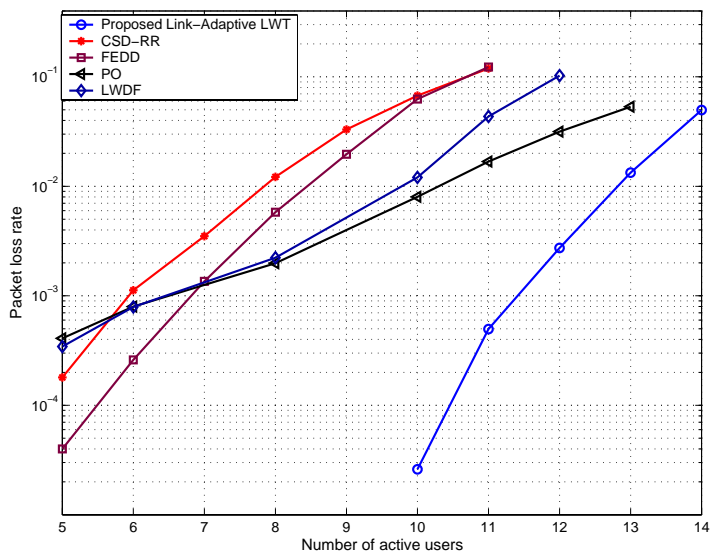

Fig. 4: Packet loss rate as a function of the number of active users. Average $E_{s} / N_{o}=22 \mathrm{~dB}$ per subcarrier.

\section{CONCLUSIONS}

In this paper, a cross layer link-adaptive LWT packet scheduling algorithm has been proposed for real-time applications in wireless packet-switched networks with OFDM signaling. The proposed algorithm endeavors to satisfy the stringent packet delay constraints of real-time traffics while obtaining a high system spectral efficiency. The algorithm is composed of two interactive components. At the MAC layer, a LWT service discipline is proposed to ensure timely and efficient delivery of the packets. Meanwhile, PHY-layer link adaptation is adopted to maximize the per-link throughput and guarantee a sufficiently low packet error rate over the wireless media. It is demonstrated in the numerical results that the proposed algorithm significantly improves the system performance in terms of spectral efficiency and packet loss rate.

\section{REFERENCES:}

[1]. H. Zhang, "Service disciplines for guaranteed performance services in packet-switching networks," IEEE Proc., vol. 83, no. 10, pp. 1374-1396, Oct. 1995.

[2]. Y. Cao and V. O. K. Li, "Scheduling algorithms in broad-band wireless networks,” IEEE Proc., vol. 89, no. 1, pp. 76-87, Jan. 2001.

[3]. S. Lu and V. Bharghavan, "Fair queueing in wireless packet networks,” IEEE/ACM Trans. Networking, vol. 7, no. 4, pp. 437-489, 1999.

[4]. P. Ramanathan and P. Agrawal, “Adapting packet fair queueing algorithms to wireless networks," Proc. ACM/IEEE MOBICOM'98, pp. 1-9, 1998.

[5]. X. Liu, E. K. P. Chong, and N. B. Shroff, "Opportunistic transmission scheduling with resource-sharing constraints in wireless networks," IEEE J. Select. Areas Commun., vol. 19, no. 10, pp. 2053-2064, 2001.

[6]. M. Andrews, K. Kumaran, K. Ramanan, A. Stolyar, P. Whiting, and R. Vijaykumar, "Providing quality of service over a shared wireless link," IEEE Commun. Mag., vol. 39, no. 2, pp. 150-154, 2001.

[7]. Y. Liu, S. Cruhl, and E. W. Knightly, "WCFQ: An opportunistic wireless scheduler with statistical fairness bounds,” IEEE Trans. Wireless Commun., vol. 2, no. 5, pp. 1017-1028, 2003.

[8]. P. Y. Kong and K. H. The, "Performance of proactive earliest due date packet scheduling in wireless networks," IEEE Trans. Vehi. Tech., vol. 53, no. 4, pp. 1224-1234, 2004.

[9]. S. T. Chung and A. J. Goldsmith, "Degrees of freedom in adaptive modulation: a unified view”, IEEE Trans. Commun., vol. 49, no. 9, pp. 1561-1571, Sept. 2001.

[10]. B. Yang, K. B. Letaief, R. S. Cheng, and Z. Cao, "Channel estimation for OFDM transmission in multipath fading channels based on parametric channel modeling," IEEE Trans. Commun., vol. 49, No. 3, March 2001.

[11]. J. G. Proakis, Digital Communications, $4^{\text {th }}$ Ed., McGraw-Hill, New York, 2001.

[12]. P. Bhagwat, A. Krishna, and S. Tripathi, "Enhancing throughput over wireless LAN's using channel state dependent packet scheduling," IEEE Proc. INFOCOM'98, pp. 1103-1111, March 1998.

[13]. S. Shakkottai and R. Srikant, "Scheduling real-time traffic with deadlines over a wireless channel," ACM/Baltzer Wireless Networks, vol. 8, no. 1, pp. 13-26, 2002. 